\title{
Investigating the Deoxyribonuclease Activity of CRM197 with Site-Directed Mutagenesis
}

Nathalie Bravo-Bautista1

Hieu Hoang ${ }^{2}$

Anusha Patel ${ }^{3}$

Jennifer Travis ${ }^{1}$

Melissa Wooten ${ }^{3}$

Nathan J. Wymer ${ }^{1, *}$

${ }^{1}$ Department of Chemistry and Biochemistry

${ }^{2}$ Department of Biological and Biomedical Sciences

${ }^{3}$ Department of Pharmaceutical Sciences

North Carolina Central University, Durham, NC 27707

Supplemental Information:

\begin{tabular}{|l|l|}
\hline \multicolumn{1}{|c|}{ Mutation } & \multicolumn{1}{c|}{ Forward Primer } \\
\hline S109A & $\begin{array}{l}\text { CAA AAA AGA ACT GGG CTT GGC CCT GAC CGA GCC } \\
\text { GCT GAT GG }\end{array}$ \\
\hline TA111A AAC TGG GCT TGA GCC TGG CCG AGC CGC TGA \\
TGG AAC AGG \\
\hline E112Q & $\begin{array}{l}\text { GAA CTG GGC TTG AGC CTG ACC CAG CCG CTG ATG } \\
\text { GAA CAG GTC GG }\end{array}$ \\
\hline S109A/T111A & $\begin{array}{l}\text { GAA CTG GGC TTG GCC CTG GCC GAG CCG CTG ATG } \\
\text { GAA CAG GTC }\end{array}$ \\
\hline S109A/E112Q & $\begin{array}{l}\text { GAA CTG GGC TTG GCC CTG ACC CAG CCG CTG ATG } \\
\text { GAA CAG GTC }\end{array}$ \\
\hline T111A/E112Q & $\begin{array}{l}\text { GAA CTG GGC TTG AGC CTG GCC CAG CCG CTG ATG } \\
\text { GAA CAG GTC }\end{array}$ \\
\hline S109A/T111A/E112Q & $\begin{array}{l}\text { GAA CTG GGC TTG GCC CTG GCC CAG CCG CTG ATG } \\
\text { GAA CAG GTC }\end{array}$ \\
\hline K103A & $\begin{array}{l}\text { GTT GAT AAT GCC GAG ACT ATC GCA AAA GAA CTG } \\
\text { GGC TTG }\end{array}$ \\
\hline E116A & $\begin{array}{l}\text { CCG CTG ATG GCA CAG GTC GGT ACT GAA GAA TTT } \\
\text { ATC AAG CGC TTC }\end{array}$ \\
\hline T120A & $\begin{array}{l}\text { GGA ACA GGT CGG TAC TGA AGC ATT TAT CAA GCG } \\
\text { CTT CGG TGA C }\end{array}$ \\
\hline E122A & $\begin{array}{l}\text { GAA CAG GTC GGT ACT GAA GAA GCT ATC AAG CGC } \\
\text { TTC GGT GAC GG }\end{array}$ \\
\hline F123A & \begin{tabular}{l} 
GTC GGT ACT GAA GAA TTT ATC GCG CGC TTC GGT \\
GAC GGT GCA AGC \\
\hline KCA CAG GTC GGT ACT GAA GCA TTT ATC AAG CGC \\
TTC GG
\end{tabular} \\
\hline R126A &
\end{tabular}




\begin{tabular}{|l|l|}
\hline E116A/E122A & $\begin{array}{l}\text { GAA CAG GTC GGT ACT GAA GCA TTT ATC AAG GCC } \\
\text { TTC GG }\end{array}$ \\
\hline E122A/R126A & $\begin{array}{l}\text { GCA CAG GTC GGT ACT GAA GCA TTT ATC AAG GCC } \\
\text { TTC GG }\end{array}$ \\
\hline E116A/E122A/R126A & $\begin{array}{l}\text { GCA CAG GTC GGT ACT GAA GCA TTT ATC AAG GCC } \\
\text { TTC GG }\end{array}$ \\
\hline
\end{tabular}

Table S1: List of forward primers used to generate CRM197 mutants using QuickChange mutagenesis protocol. 